\title{
The metabolic effects of TNF inhibitors in rheumatoid arthritis patients
}

\author{
Alexandra Jitaru ${ }^{1,2}$, Maria Magdalena Leon-Constantin ${ }^{1,3}$, Cristina Pomirleanu ${ }^{2,4}$, \\ Codrina Ancuta ${ }^{2,4}$, Florin Mitu ${ }^{1,3}$ \\ ${ }^{1}$ Department of Medical Semiology, "Grigore T. Popa” University of Medicine and Pharmacy, Iasi, Romania \\ 22nd Rheumatology Department, Clinical Rehabilitation Hospital, Iasi, Romania \\ ${ }^{3}$ Cardiovascular Rehabilitation Department, Clinical Rehabilitation Hospital, Iasi, Romania \\ ${ }^{4}$ Department of Rheumatology and Rehabilitation, "Grigore T. Popa” University of Medicine and Pharmacy, \\ lasi, Romania
}

\begin{abstract}
Objective. Cardiovascular disease (CVD) is considered to be the main cause of morbidity and mortality in rheumatoid arthritis (RA) patients. Tumor necrosis factor alpha (TNF), an important pro-inflammatory cytokine, has a multivalent role in the pathogenesis of RA. It is also responsible for the glucose metabolism related alterations and for the increased levels of serum lipids found in these patients. We aimed to evaluate the effects of TNF inhibitors on lipid and glucose metabolism in patients with active RA.

Methods. We performed a prospective study in 117 consecutive patients with active RA undergoing periodic treatment with TNF inhibitors - Adalimumab (ADA), Etanercept (ETA), Infliximab (IFX) -, combined with conventional synthetic disease modifying antirheumatic drugs (csDMARDs), according to the national recommendations for the treatment of RA. At the time of the enrollment, all patients were assessed according to a predefined protocol including demographics and RA-related parameters (inflammatory syndrome, disease activity, medication) as well as metabolic biomarkers such as total cholesterol (TC), HDL cholesterol (HDL-C), LDL cholesterol (LDL-C), triglycerides (TG) and serum glucose level. Statistical analysis was performed in STATA 12/SE software, $p<0.05$. Results. We demonstrated a significant decrease in TC, TG and fasting glucose levels, particularly in patients treated with ADA and ETA $(p<0.05)$. Moreover, we noticed a significant correlation between the mean levels of lipid and glucose and inflammatory parameters (ESR and CRP), but not with DAS28-CRP. The same strong correlation was demonstrated between the decrease of TC and TG values ( $\triangle T C$ and $\Delta T G$, respectively) and the decrease of CRP and ESR values ( $\triangle \mathrm{CRP}$ and $\triangle E S R$, respectively), that is not maintained with DAS28-CRP score. Conclusions. TNF inhibitors, particularly ADA and ETA are able to decrease serum levels of both lipid and glucose metabolism in patients with active RA in long-term administration, indirectly modulating the cardio-vascular risk.
\end{abstract}

Keywords: rheumatoid arthritis, lipid markers, TNF inhibitors, glucose level

\section{INTRODUCTION}

Cardiovascular disease (CVD) is considered to be the main cause of morbidity and mortality in rheumatoid arthritis (RA) patients, the risk of death being up to $50 \%$ bigger than in general population [1]. Some of the drivers of these statistical data are the metabolic changes associated with the disease itself and the medication [2]. Many studies showed an increased prevalence of metabolic syndrome among patients with RA, with differences between studies, depending on the different assessment criteria, disease duration and activity [3]. Tumor necrosis factor alpha (TNF), an important pro-inflammatory cytokine, has a multivalent role in the pathogenesis of RA. It is also responsible for the glucose metabolism related alterations and for the increased levels of serum lipids found in these patients [4]. Despite the increased risk of CVD, the prevalence of dyslipidemia in RA patients is not significantly different from the general population [5]. Recent studies reveal that, in early RA, the levels of total cholesterol (TC) and LDL cholesterol (LDL-C) are decreased, while other studies demonstrate a high level of TC, LDL and HDL cholesterol (HDL-C) [6]. 
TNF inhibitors - Adalimumab (ADA), Etanercept (ETA), Infliximab (IFX), Golimumab (GLM) and Certolizumab pegol (CZM) - are known to reduce disease activity and improve patient quality of life in RA [7]. However, their impact on the cardiovascular risk remains controversial, especially related to the lipid and glucose metabolism. Recent studies of lipid profiles in RA patients reveal that TNF inhibitors and interleukin-6 receptor (IL-6R) inhibitors, in monotherapy, worsen lipid and glucose levels, while combination therapy (conventional synthetic and biological agents) has a positive impact, especially when biological agents are associated with hydroxychloroquine (8).

Our study aimed to evaluate the effects of TNF inhibitors on metabolic parameters (serum lipids and glucose) in patients with active RA and to identify corelations between metabolic and inflammatory biomarkers as well as disease activity.

\section{MATERIAL AND METHODS}

\section{Patient population}

We assessed a cohort of 117 consecutive patients with RA undergoing periodic treatment with TNF inhibitors (ADA, ETA, IFX) as their first biologic, combined with conventional synthetic disease modifying anti-rheumatic drugs (csDMARDs), according to the National Protocol and European League Against Rheumatism (EULAR) recommendations for the treatment of RA $[9,10]$. They were recruited from the Outpatient Rheumatology Department, Clinical Rehabilitation Hospital in Iasi and followed-up for a period of 3 years (01.01.2016 - 31.12.2018). All patients were 18 years or older and fulfilled the 1987 and 2010 classification criteria for RA $[11,12]$. Also, they had preexistent CVD (arterial hypertension, coronary disease, heart failure, stroke). Based on their TNF inhibitor, the participants were divided in 3 subgroups as follows: 51 patients treated with ADA, 38 patients treated with ETA and 28 receiving IFX.

At the time of enrollment, all patients were assessed according to the same complex protocol including (i) demographic parameters - age, gender, smoking status, body mass index (BMI); (ii) diseaserelated parameters - disease duration, stage, clinical (tender and swollen joint count on 28 evaluable joints, morning stiffness, pain on Visual Analog Scale (VAS), erythrocyte sedimentation rate (ESR) and $\mathrm{C}$ reactive protein (CRP), disease activity score (DAS28-CRP) and disability (HAQ-DI), concomitant medication (csDMARDs); and (iii) metabolic parameters - total cholesterol (TC) and fractions HDL cholesterol (HDL-C), LDL cholesterol (LDLC) and triglycerides (TG) and serum glucose level.

Patients with a medical history of cardiac surgery or myocardial infarction or stroke 6 months before the onset, or diagnosed with diabetes mellitus (DM) and/or dyslipidemia, as well as those undergoing corticosteroid or statin therapy were not included in the study.

ADA and ETA were administered subcutaneously at a dose of $40 \mathrm{mg}$ every 2 weeks and $50 \mathrm{mg}$ every week, respectively. IFX was administered intravenously at a dose of $3 \mathrm{mg} / \mathrm{kg}$ at 0,2 and 6 weeks, then every 8 weeks.

\section{Study protocol}

Blood samples were taken at the onset and the end of the study. Serum glucose, TC, HDL-C, LDL$\mathrm{C}$ and TG levels were measured by standard automated methods on every participant, at the onset and the end of the study (at 52 weeks). ESR was determined using the Westergren method and CRP by latex immuno-turbidimetry. The other parameters were assessed according to the national protocol (every 6 months).

The study protocol was approved by the local institutional ethics committee. All patients gave informed consent to participate in this study previous to their inclusion.

\section{Statistical analysis}

Statistical analysis was performed using STATA 12/SE (StataCorp, College Station, TX, USA). Differences statistically significant were considered at $\mathrm{p}<0.05$. Results were reported as mean \pm standard deviation (SD) and t-student test was used for the comparison of normally distributed variables between groups.

\section{RESULTS}

We assesed 117 consecutive patients with RA and concomitant CVD, undergoing TNF inhibitors and csDMARDS for their active disease. All patients were bio-naïve at the enrollment in the study and $44 \%$ received ADA, $32 \%$ received ETA and $24 \%$ received IFX. 


\section{Baseline assessment}

Baseline characteristics of the patients enrolled in the study are shown in table 1 . More than half of our patients were women $(63.38 \%)$, with mean age of $56.8 \pm 11.7$ years and mean BMI of $31.3 \pm 11.6$; the majority were classified as having established RA and had seropositive (rheumatoid factor and anticyclic citrullinated peptide antibodies) disease (88.03\%). Furthermore, all patients presented with moderate to severe active disease, with a mean DAS28-CRP of $5.88 \pm 0.8$.

About one third had arterial hypertension (31.3\%), 12.5\% coronary heart disease, $6.3 \%$ stroke, while $1.3 \%$ moderate heart failure.

Baseline serum levels of lipid parameters were above the normal range (TC $238.49 \pm 34.91 \mathrm{mg} . / \mathrm{dl}$, HDL-C $71.66 \pm 17.43 \mathrm{mg} / \mathrm{dl}$, LDL-C $146.7 \pm 28.08$ $\mathrm{mg} / \mathrm{dl}$, TG $178.3 \pm 21.5 \mathrm{mg} / \mathrm{dl}$ ), indirectly suggesting an increased cardiovascular risk among our RA patients. Also, the baseline serum glucose was higher than the normal limits (117.5 \pm 17.29$)$, as seen in table 1 .

TABLE 1. Baseline characteristics of the patients enrolled in the study

\begin{tabular}{|c|c|}
\hline Age at onset (years) & $56.08 \pm 11.76$ \\
\hline Women (\%) & $80(63.38 \%)$ \\
\hline Disease duration (months) & $140 \pm 108.48$ \\
\hline Non-smokers (\%) & $90(76.56 \%)$ \\
\hline $\mathrm{BMI}\left(\mathrm{kg} / \mathrm{m}^{2}\right)$ & $31.3 \pm 11.6$ \\
\hline Rheumatoid factor (RF) positive (n, \%) & $103(88.03 \%)$ \\
\hline $\begin{array}{l}\text { Anti-citrullinated peptide antibody } \\
\text { (ACPA) positive }(n, \%)\end{array}$ & 76 (64.95\%) \\
\hline \multicolumn{2}{|l|}{ Disease activity } \\
\hline DAS28-CRP & $5.88 \pm 0.8$ \\
\hline Tender joint count (0-28) & $6.5 \pm 7.35$ \\
\hline Swollen joint count $(0-28)$ & $6.1 \pm 4.62$ \\
\hline $\begin{array}{l}\text { Visual Analog Scale (VAS) patient } \\
\text { disease activity }\end{array}$ & $75.3 \pm 14.72$ \\
\hline $\mathrm{CRP}(\mathrm{mg} / \mathrm{l})$ & $11.47 \pm 4.92$ \\
\hline ESR (mm/1 hour) & $33.23 \pm 7.47$ \\
\hline \multicolumn{2}{|l|}{ Cardiovascular comorbidities } \\
\hline Arterial hypertension (n, \%) & $37(31.3 \%)$ \\
\hline Stroke (n, \%) & $7(6.3 \%)$ \\
\hline Coronary disease $(n, \%)$ & $15(12.5 \%)$ \\
\hline Heart failure $(n, \%)$ & $2(1.3 \%)$ \\
\hline \multicolumn{2}{|l|}{ Metabolic parameters } \\
\hline $\mathrm{TC}(\mathrm{mg} / \mathrm{dl})$ & $238.49 \pm 34.91$ \\
\hline $\mathrm{HDL}-\mathrm{C}(\mathrm{mg} / \mathrm{dl})$ & $71.66 \pm 17.43$ \\
\hline LDL-C (mg/dl) & $146.7 \pm 28.08$ \\
\hline TG $(\mathrm{mg} / \mathrm{dl})$ & $178.3 \pm 21.5$ \\
\hline Fasting glucose (mg/dl) & $117.5 \pm 17.29$ \\
\hline
\end{tabular}

\begin{tabular}{|l|c|}
\hline \multicolumn{2}{|l|}{ csDMARDs } \\
\hline Methotrexate (MTX) (n, \%) & $66(56.41 \%)$ \\
\hline Leflunomide (LEF) (n, \%) & $28(23.93 \%)$ \\
\hline Sulfasalazine (SSZ) (n, \%) & $6(5.12 \%)$ \\
\hline Biological DMARDs & $51(44 \%)$ \\
\hline ADA & $38(32 \%)$ \\
\hline ETA & $28(24 \%)$ \\
\hline IFX &
\end{tabular}

$\mathrm{BMI}$, body mass index; RF, rheumatoid factor; $\mathrm{ACPA}$, anti-citrullinated peptide antibody; DAS28-CRP, disease activity score by evaluation of 28 joints; VAS, Visual Analog Scale; CRP, C-reactive protein; ESR, erytrocytes sedimentation rate; $\mathrm{HAQ}$, Health Assessment Questionnaire; DM, diabettes mellitus; TC, total cholesterol; HDL, high density lipoprotein; LDL, low density lipoprotein; TG, triglycerides; csDMARDs, conventional synthetic Disease Modifying Anti Rheumatic Drugs; MTX, Methotrexate; LEF, Leflunomide; SSZ, Sulfasalazine; ADA, Adalimumab; ETA, Etanercept; IFX, Infliximab

\section{2-weeks assessments}

Globally, we reported significant improvement in all clinical and lab parameters as well as disease activity score at 52 weeks of treatment, the majority of patients reaching moderate or even low disease activity as supported by DAS28-CRP, indicating drug efficacy; also subgroup analysis showed significant improvement of disease activity as compared to baseline (table 2).

We also noticed a significant decrease of the mean levels of lipid parameters as well as serum glucose at the final evaluation, as seen in table 2 .

Subgroup analysis showed a decrease in TC, TG and fasting glucose levels in patients treated with ADA (figure 1): both TC and TG values decreased significantly, the difference between the 2 graphical curves being relatively constant.

Among the patients treated with ETA, there is also a decrease in the values of TC, TG and glucose. Similar to ADA, a significant decrease is recorded especially for the TC and TG values, the distance between the 2 graph lines being relatively constant (figure 2).

For the particular case of IFX treatment, a decrease in TC, TG and glucose levels is generally observed; however, it is easy to notice that the decrease of the serum levels of metabolic parameters is significant only for some patients, while in others it is negligible or there even increased (figure 3 ).

A strong correlation was observed between the decrease of TC and TG values $(\Delta T C$ and $\Delta T G$, respectively) and the decrease of CRP and ESR values ( $\triangle \mathrm{CRP}$ and $\triangle \mathrm{ESR}$, respectively), illustrated by the trend of polynomial curves of the values recorded among the patients; there is also a weaker correlation 
TABLE 2. Evolution of the disease activity and metabolic parameters in each study subgroup

\begin{tabular}{|l|c|c|c|c|c|c|}
\hline Parameters & \multicolumn{2}{|c|}{ ADA } & \multicolumn{2}{c|}{ ETA } & IFX \\
\hline Disease activity & Initial & Final & Initial & Final & Initial & Final \\
\hline DAS28-CRP & $5.8 \pm 0.8$ & $2.78 \pm 0.75$ & $5.95 \pm 0.89$ & $2.79 \pm 0.68$ & $5.9 \pm 0.87$ & $2.98 \pm 2.8$ \\
\hline CRP $(\mathrm{md} / \mathrm{dl})$ & $11.78 \pm 5.21$ & $2.71 \pm 1.81$ & $11.92 \pm 4.45$ & $2.34 \pm 1.69$ & $10.29 \pm 4.82$ & $2.75 \pm 1.72$ \\
\hline ESR (mm/1h) & $34 \pm 7.07$ & $21.5 \pm 6.24$ & $31.74 \pm 7.26$ & $20.58 \pm 6.7$ & $32.86 \pm 8.03$ & $25.25 \pm 7.73$ \\
\hline $\begin{array}{l}\text { Patient global } \\
\text { assessment 0-100 }\end{array}$ & $74.55 \pm 14$ & $54.51 \pm 9$ & $78.08 \pm 14.8$ & $54.71 \pm 8.9$ & $72.93 \pm 15.32$ & $65.32 \pm 7.86$ \\
\hline Metabolic markers & \multicolumn{7}{|c|}{} & & & \\
\hline TC (mg/dl) & $238.51 \pm 31.47$ & $206.57 \pm 14.38$ & $240.32 \pm 37.03$ & $201.55 \pm 14.79$ & $236 \pm 37.68$ & $220.79 \pm 15.21$ \\
\hline HDL-C (mg/dl) & $68.59 \pm 17.47$ & $55.82 \pm 8.86$ & $77.13 \pm 17.07$ & $55.21 \pm 8.98$ & $69.86 \pm 16.05$ & $61.57 \pm 9.24$ \\
\hline LDL-C (mg/dl) & $148.14 \pm 26.82$ & $118.39 \pm 12.33$ & $145 \pm 31.13$ & $120 \pm 13.22$ & $146.43 \pm 25.78$ & $132.11 \pm 14.77$ \\
\hline TG (mg/dl) & $175.51 \pm 22.43$ & $153.8 \pm 15.76$ & $181.26 \pm 21.28$ & $151.95 \pm 17.14$ & $179.64 \pm 19.56$ & $164.25 \pm 18.53$ \\
\hline GLC (mg/dl) & $118.61 \pm 17.89$ & $102.45 \pm 8.67$ & $119.95 \pm 16.19$ & $98.97 \pm 10.02$ & $112.93 \pm 16.73$ & $102.75 \pm 10.37$ \\
\hline
\end{tabular}

DAS28, Disease Activity Score; CRP, C-reactive protein; ESR, erythrocyte sedimentation rate; VAS, Visual Analog Scale; TC, total cholesterol; HDL, high density lipoprotein; LDL, low density lipoprotein; TG, triglycerides; GLC, serum glucose

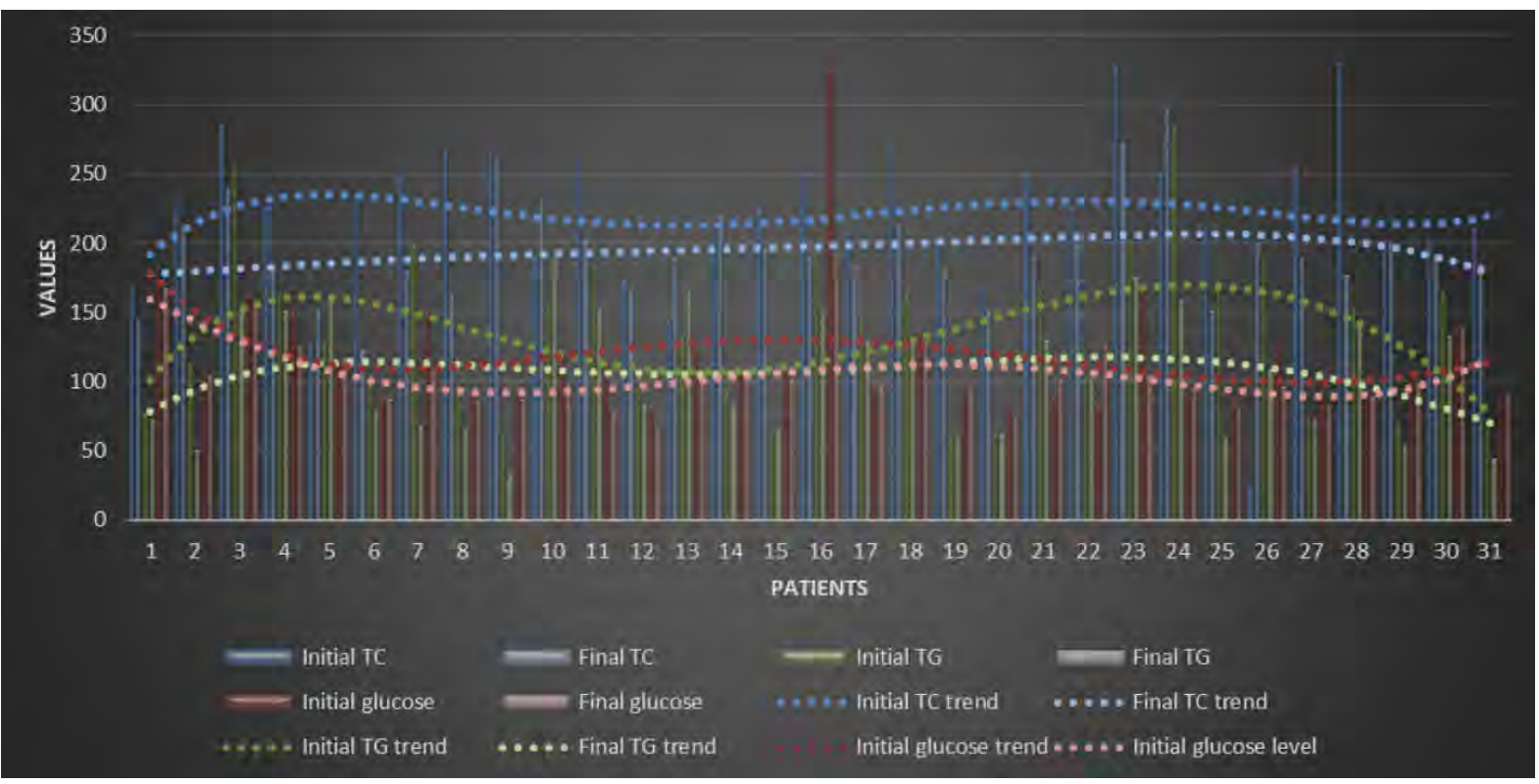

FIGURE 1. Evolution of TC, TG and glucose values in patients undergoing ADA therapy (graphically illustrated by the areas delimited by the initial and final polynomial trend)

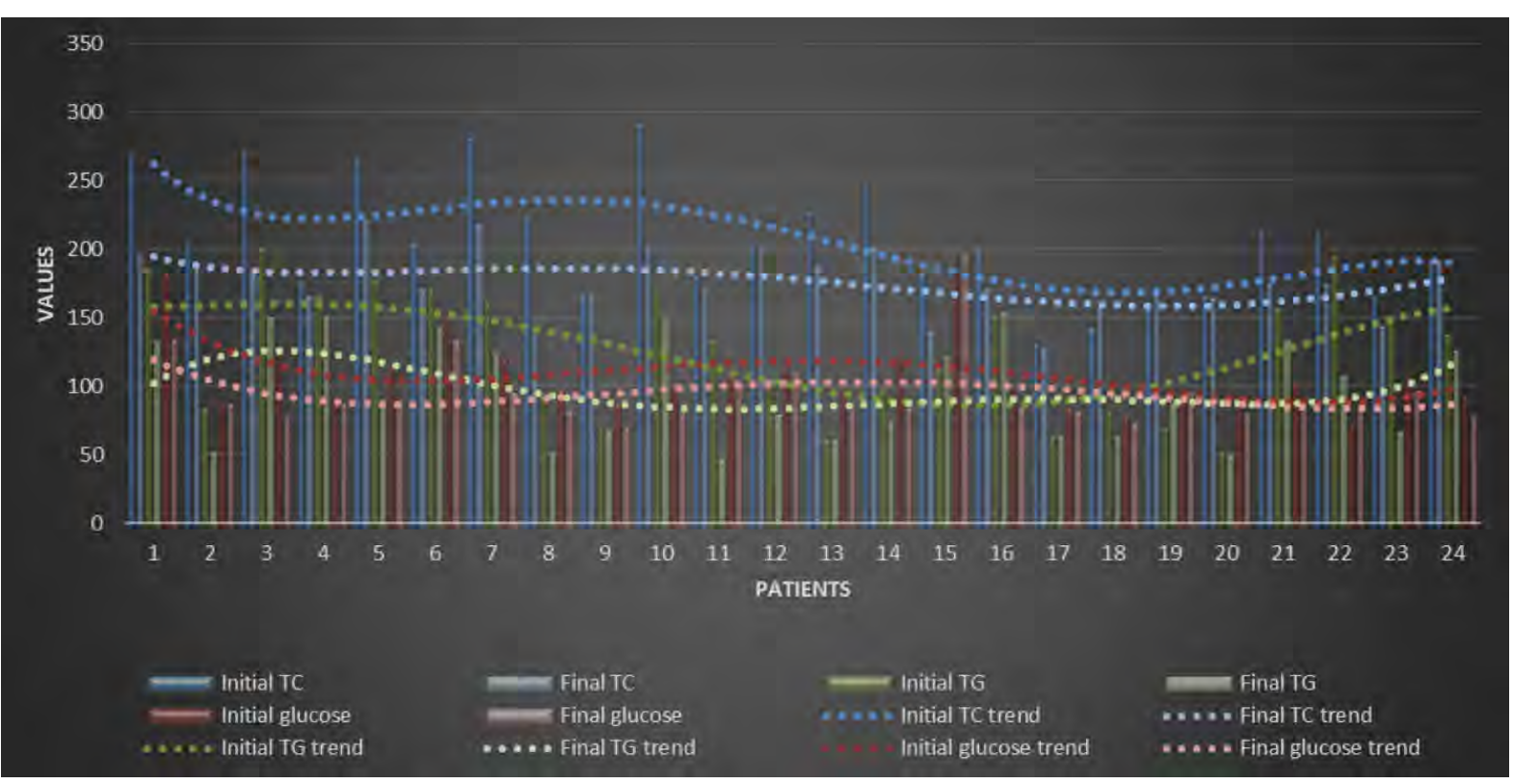

FIGURE 2. Evolution of TC, TG and glucose values in patients undergoing ETA therapy (graphically illustrated by the areas delimited by the initial and the final trend) 


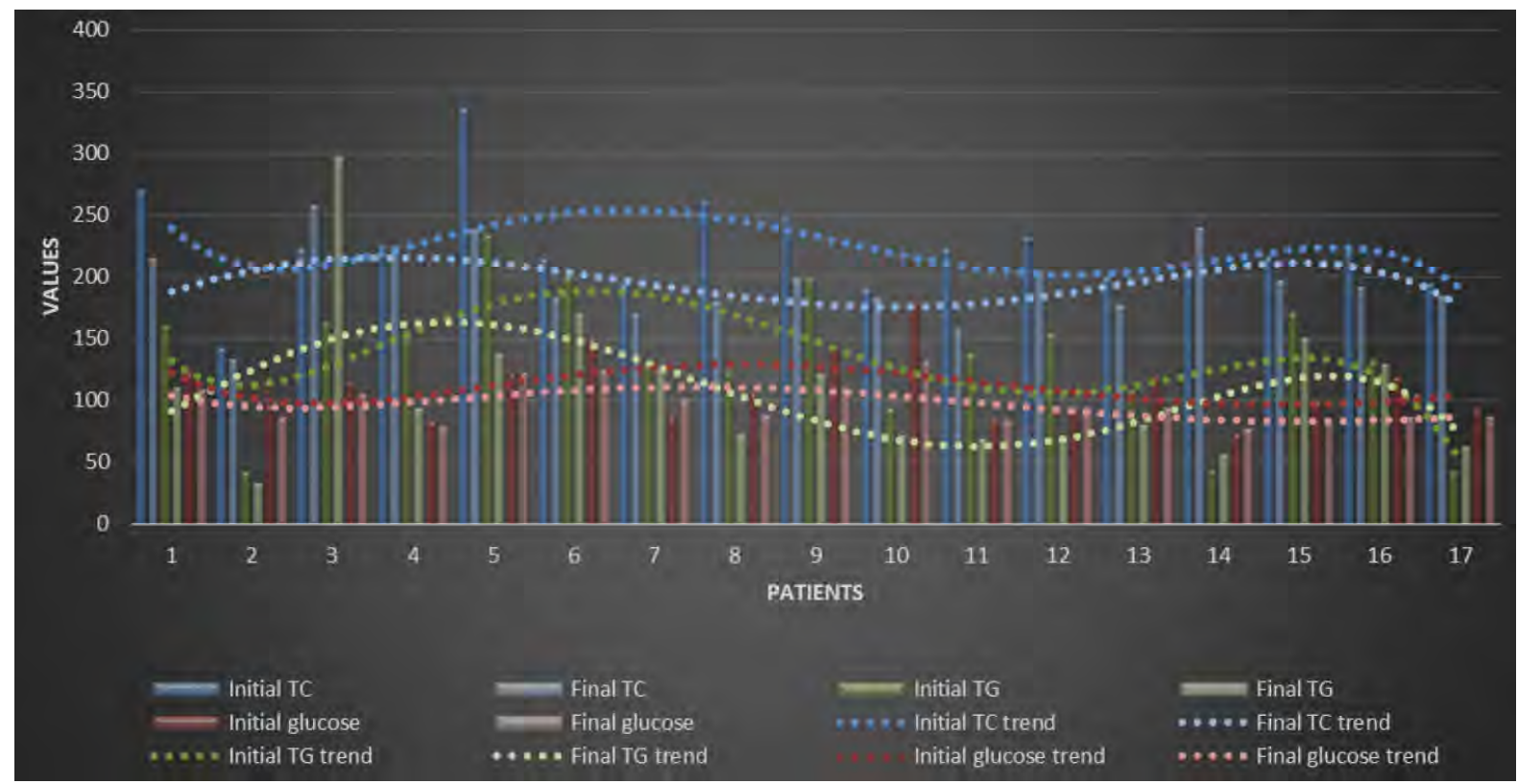

FIGURE 3. Evolution of TC, TG and glucose values in patients undergoing IFX therapy (illustrated graphically by the areas delimited by the initial and final polynomial trend)

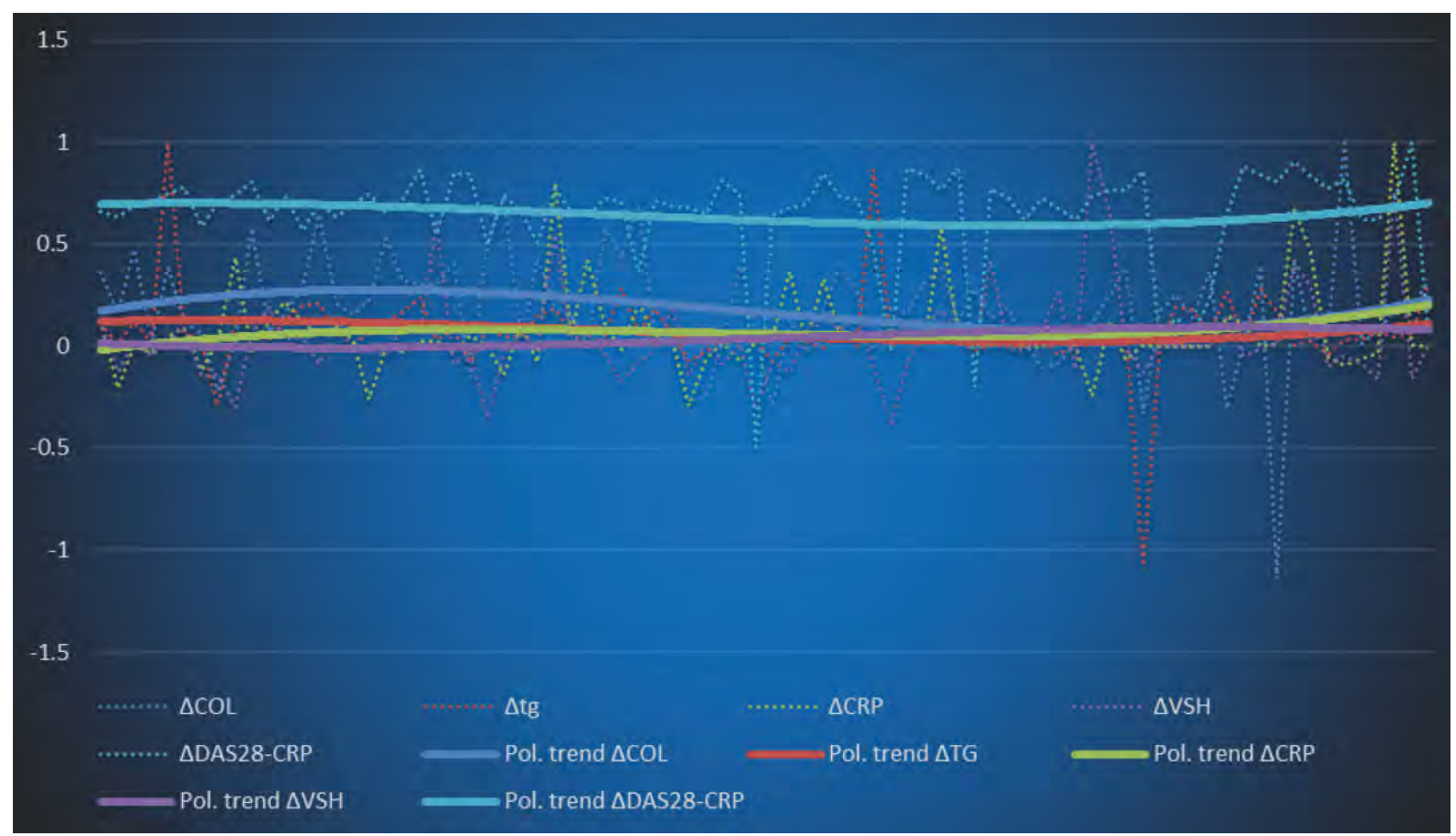

FIGURE 4. The correlation between $\triangle C R P, E S R, T C, T G$ and glucose and normalized DAS28-CRP

with the decrease of the DAS28-CRP score, suggested by a similar trend, but with a higher error (figure 4).

\section{DISCUSSION}

We performed a study on a significant cohort of active RA patients, presenting different disease subtypes (seropositive, seronegative), with concomitant CVD, receiving TNF inhibitors combined with csDMARDs for 52 weeks. We assessed the impact of the medication on metabolic parameters (lipids and serum glucose) and, indirectly on the cardiovascular risk. Our results clearly demonstrated the dynamic decrease of these parameters, both globally and in subgroup analysis, particularly in patients under ADA and ETA therapy. Furthermore, the study the study showed an important correlation with inflammatory syndrome, but not with the composite score of disease activity.

The results obtained are consistent with the data from the literature, especially regarding the treat- 
ment with IFX. Concerning the treatment with ADA and ETA, the data are contradictory; there are studies that show a less significant impact of the therapy with biological agents on the lipid and glucose metabolism as compared to our results.

Different studies have already evaluated the cardiovascular safety of TNF $\alpha$ inhibitors and other nonTNF drugs as well as their influence on cardiovascular risk factors in patients with active RA. Nurmohamed et al. [13] evaluated the impact of individual TNF inhibitors on different risk factors; they concluded that ADA treatment did not show any effect on TC or TG values, while HDL cholesterol and LDL cholesterol showed slight increases. Moreover, ADA therapy resulted in a significant reduction in CRP level. The myocardial infarction rate was $0.3-0.6 \%$, while the stroke rate was $0.3 \%$, and the incidence of thromboembolic events was higher in patients with immunogenicity phenomena, especially in those who developed anti-ADA antibodies (2.5\%), unlike those without these modifications $(0.8 \%)$. Regarding the administration of ETA, an increase of HDL-C was observed, without significant effects on LDL-C and TG. There were also marked decreases in CRP and DAS28-CRP values, as well as a low rate of major cardiovascular events (1\%). However, in patients with diabetes mellitus and/or chronic lung disease, the rate of these complications was higher. Data about IFX were quite controversial, but consistent with those obtained in our research. Thus, increases in TC, HDL-C and TG values were observed along with a less significant effect on LDL$\mathrm{C}$; serum CRP levels remained low during IFX therapy, as well as the rate of major cardiovascular events such as myocardial infarction or stroke [13].

An interesting paper regarding the association between dyslipidemia and inflammation in RA was a case-control study published in 2018 by Clarke et al.; they assessed 1905 patients highlighting the important correlation between pro-inflammatory cytokines and the modification of lipid biomarkers. Although TNF $\alpha$ is directly involved in this phenomenon, significant results were also obtained in relation to IL-6, this cytokine having the strongest association with cardiovascular disease. As a result, the level of IFN $\gamma$, which is known to be modulated by IL-6, is directly proportional to cardiovascular risk. Thus, after modulation of IL-6 levels, the association between IFN- $\gamma$ and CVD is significantly decreased [14].

It is important to mentioned another longitudinal cohort study, carried out by Audrey et al. and published in 2017 over a period of 5 years and including 6902 patients with active RA, demonstrating that TNF inhibitors are associated with a lower cardiovascular risk, compared to the synthetic immunosuppressive therapy; moreover, $\mathrm{TNF} \alpha$ inhibitors have a higher protective effect than IL-12/23 inhibitors [15].

Finally, we can conclude that RA patients undergoing TNF inhibitors therapy had an indirect reduction of cardiovascular risk.

Returning to our study, we consider that our results are relevant for routine medical practice in Romania; indeed, the study has several limits that are worth mentioning: the short duration of follow-up, a rather classic than extensive evaluation of lipid metabolism, as well as the absence of a control group. Further studies are needed in larger cohorts of RA patients, with a more complex evaluation of the metabolic parameters (including apolipoprotein A, glycated hemoglobin) not only related to TNF inhibitors, but also to other biological DMARDs. We also consider relevant to assess other disease activity scores (SDAI, CDAI) than DAS28-CRP and their relation with lipid and glucose metabolism.

\section{CONCLUSIONS}

The result of our study demonstrated that the levels of TC, TG and glucose directly correlate with disease activity in RA patients (DAS28-CRP, ESR, CRP). Moreover, the TNF $\alpha$ blockade is associated with lower cardiovascular risk in these patients.

Conflict of interest: none declared Financial support: none declared

\section{REFERENCES}

1. Mavrogeni S, Dimitroulas T, Bucciarelli-Ducci $C$ et al. Rheumatoid arthritis: An autoimmune disease with female preponderance and cardiovascular risk equivalent to diabetes mellitus: role of cardiovascular magnetic resonance. Inflamm Allergy DrugTargets. 2014; 13(2):81-93

2. Corradoa $\mathrm{A}$, Coliaa $\mathrm{R}$, Rotondo $\mathrm{C}$ et al. Changes in serum adipokines profile and insulin resistance in patients with rheumatoid arthritis treated with anti-TNF-a. Curr Med Res Opin. 2019; 35(12):2197-2205.

3. Liao KP. Cardiovascular disease in patients with rheumatoid arthritis. Trends Cardiovasc Med. 2017;27(2): 136-140.

4. Barbhaiya M, Solomon DH. Rheumatoid arthritis and cardiovascular disease: An update on treatment issues. CurrOpinRheumatol. 2013;25(3):317-24. 
5. Atzeni F, Gianturco L, Boccassini L et al. Noninvasive imaging methods for evaluating cardiovascular involvement in patients with rheumatoid arthritis before and after anti-TNF drug treatment. Future Sci OA. 2019 Jun 13;5(6): FSO396.

6. Pašková U. Lipid profile and risks of cardiovascular diseases in conditions of rheumatoid arthritis. CeskaSlov Farm. 2019; 68(6):219-228

7. Deodhar A, Bitman B, Yang Y, Collier DH. The effect of etanercept on traditional metabolic risk factors for cardiovascular disease in patients with rheumatoid arthritis. Clin Rheumatol. 2016; 35(12):3045-3052.

8. Nicolau J, Lequerré $\mathrm{T}$, Bacquet $\mathrm{H}$, Vittecoq $\mathrm{O}$. Rheumatoid arthritis, insulin resistance, and diabetes. Joint Bone Spine. 2017 Jul; 84(4):411-416

9. Nam JL, Takase-Minegishi K, Ramiro S et al. Efficacy of biological disease-modifying antirheumatic drugs: a systematic literature review informing the 2016 update of the EULAR recommendations for the management of rheumatoid arthritis. Ann Rheum Dis. 2017 Jun; 76(6):1113-1136.

10. ${ }^{* \star *}$ Protocol terapeutic in artrita reumatoida privind utilizarea agentilor biologici adalimumabum (original si biosimilar), certolizumabum, etanerceptum (original si biosimilar) golimumabum, infliximabum (original si biosimilar), dec 2019, pg 164-180 file:///C:/Users/HP/Downloads/Lista\%20 protocoalelor\%20decembrie-\%202019.pdf

11. Arnett FC, Edworthy SM, Bloch DA et. al. The American Rheumatism Association 1987 revised criteria for the classification of rheumatoid arthritis. Arthritis Rheum. 1988 Mar;31(3):315-24.

12. Kay J, Upchurch KS. ACR/EULAR 2010 rheumatoid arthritis classification criteria. Rheumatology 2012; 51:vi5vi9 doi:10.1093/ rheumatology/kes279.

13. Nurmohamed M, Choy E, Lula S et al. The Impact of Biologics and Tofacitinib on Cardiovascular Risk Factors and Outcomes in Patients with Rheumatic Disease: A Systematic Literature Review. Drug Saf. 2018; 41:473-488.

14. Clarke R, Valdes-Marquez E, Hill M et al. Plasma cytokines and risk of coronary heart disease in the PROCARDIS study. Open Heart. 2018; 5(1): e000807.

15. Audrey S L Low et al. Relationship between exposure to tumour necrosis factor inhibitor therapy and incidence and severity of myocardial infarction in patients with rheumatoid arthritis. Ann Rheum Dis. 2017;76(4): 654-660. 